ORIGINAL ARTICLE

\title{
The immunohistochemical localisation of somatostatin receptors 1, 2, 3, and 5 in acoustic neuromas
}

\author{
N D Stafford, L T Condon, M J C Rogers, L Helboe, D A Crooks, S L Atkin
}

J Clin Pathol 2004;57:168-171

See end of article for authors' affiliations .....................

Correspondence to: Professor N D Stafford Postgraduate Medical Institute, Cohen Building, Room 267, The University of Hull, Cottingham Road, Hull HU6 7RX, UK;

N.D.Stafford@hull.ac.uk

Accepted for publication 7 April 2003

\begin{abstract}
Aims: Acoustic neuroma is a benign tumour, which develops through an overproliferation of Schwann cells along the vestibular nerve. Somatostatin is a naturally occurring peptide, which exerts antiproliferative and antiangiogenic effects via five membrane bound receptor subtypes. The aim of this study was to determine whether somatostatin receptor subtypes (SSTRs) 1, 2, 3, and 5 are present in acoustic neuromas.

Methods: The expression of SSTRs 1, 2, 3, and 5 was studied in both the Schwann cells and blood vessels of eight acoustic neuroma specimens, by means of immunohistochemistry using novel rabbit polyclonal antibodies raised against human SSTR 1,2, and 5 subtype specific peptides, and a commercial antiSSTR3 antibody.

Results: SSTR2 was the most prevalent subtype in Schwann cells (seven of eight), with intermediate expression of SSTR3 (six of eight), and lower expression of SSTRs 1 and 5 (four of eight and five of eight, respectively). There was ubiquitous vascular expression of SSTR2, with no evidence of SSTR 1, 3, or 5 expression in blood vessels.

Conclusion: SSTRs 1, 2, 3, and 5 are differentially expressed in acoustic neuromas. Somatostatin analogues may have a therapeutic role in the management of this rare and challenging condition.
\end{abstract}

A coustic neuroma is a rare $(8 \%$ of primary intracranial tumours) and therapeutically challenging condition, which arises from an overproliferation of Schwann cells along the vestibular nerve. Ninety per cent of acoustic neuromas are unilateral and sporadic, whereas the remaining $10 \%$ are bilateral and hereditary. The latter occur in patients with neurofibromatosis type 2, an autosomal recessive disorder, caused by functional inactivation of both copies of the NF2 gene on chromosome 22, and associated with other abnormalities (such as neurofibromas and multiple meningiomas). ${ }^{1}$ Acoustic neuromas are well circumscribed and encapsulated, with the nerve fibres splayed out across their surface, and microscopically contain regions of high and low cellularity known as Antoni A and Antoni B areas, respectively. $^{2}$

Somatostatin was discovered in 1973 as an inhibitory hypothalamic neuropeptide and called somatotroph release inhibiting factor (SRIF). ${ }^{3}$ This peptide hormone is found throughout the body, where it inhibits several physiological processes, such as endocrine and exocrine pancreatic secretion, neurotransmission in the central nervous system, and gut motility. ${ }^{4}$ The inhibitory effects of SRIF on cell proliferation and angiogenesis are of particular interest in the field of oncology. Indeed, SRIF has been dubbed "the body's naturally occurring inhibitory peptide ${ }^{\prime \prime} .{ }^{5}$ This has led to the development of SRIF analogues, which are established treatments in several neuroendocrine tumours, ${ }^{65}$ and are undergoing phase II clinical trials in other malignant conditions. $^{7}$

SRIF is a small peptide, which exists in two bioactive forms, consisting of 14 and 28 amino acids. SRIF acts on target cells via a superfamily of five membrane bound receptors, all of which have been cloned. ${ }^{8}$ Each receptor subtype consists of seven transmembrane spanning domains, with an intracellular C-terminal, which is coupled to a G protein. This $G$ protein is in turn linked to multiple intracellular transduction pathways, such as the inhibition of adenylate cyclase, the activation of $\mathrm{K}^{+}$channels, and the activation of tyrosine phosphatases. ' This last activity correlates with the antiproliferative effects of SRIF, particularly via somatostatin receptor 2 (SSTR2), and leads to G1 cell cycle growth arrest. ${ }^{10}$

"All five somatostatin receptors have an antiproliferative effect, either by inhibition of mitogenesis or stimulation of apoptosis"

Many normal and malignant cell types express multiple receptor subtypes. Despite this complexity, studies using receptor subtype specific analogues, and more recently animal "knockout" models with selective receptor subtype deletions, have enabled specific functions to be attributed to each of the SRIF receptors. All five SSTRs have an antiproliferative effect, either by inhibition of mitogenesis or stimulation of apoptosis. SSTRs 1, 2, 4, and 5 induce G1 cell cycle growth arrest, whereas receptor 3 is proapoptotic, via the induction of p53 and BAX. ${ }^{11}{ }^{12}$ SSTRs 2,3 , and 5 are also antiangiogenic. ${ }^{13}$

SRIF has a short half life of around 90 seconds, hence the development of synthetic analogues, such as SMS-201-905 (Sandostatin, octreotide), RC-160 (Vaproetide, octastatin), and BIM-23014 (Lanreotide, somatuline), which have varying binding affinities for the different receptor subtypes. These drugs are administered parenterally, their peptide nature precluding oral administration. SRIF analogues are very safe; gallbladder "sludge" has been reported in $20 \%$ and gallstones in a further $20 \%$ of a cohort of 103 patients, 12 months after beginning treatment with octreotide for acromegaly. ${ }^{14}$

Abbreviations: BSA, bovine serum albumin; HRP-StrepABC, horseradish peroxidase conjugated streptavidin-biotin complex; PBS, phosphate buffered saline; SRIF, somatotroph release inhibiting factor; SSTR, somatostatin receptor 
The administration and subsequent scintigraphic localisation of a radiolabelled somatostatin analogue (for example, Octreoscan ${ }^{\circledR}$ ) is an established technique, which allows tumour SSTR status to be determined in vivo. ${ }^{15} 16$ It is primarily used to assess disease extent and the presence of metastases in neuroendocrine tumours, and can be used to monitor response to treatment.

The aim of our study was to define the expression of SSTRs $1,2,3$, and 5 in a series of acoustic neuromas, to indicate whether SRIF analogues may have a potential therapeutic role in this condition. Therefore, immunohistochemical analysis was undertaken, using novel polyclonal antibodies to SSTRs 1, 2, and 5, and a commercial antibody to receptor 3.

\section{MATERIALS AND METHODS}

Local ethics committee permission was obtained to access material from the pathology archives at Hull and East Yorkshire NHS Trust (Hull, UK). The pathology specimens of all eight patients treated surgically for acoustic neuroma at Hull Royal Infirmary between 1995 and 2000 were retrieved. Five of the patients were men, three were women, and the mean age at diagnosis was 45 (range, 22-74). None of the patients had von Recklinghausen's syndrome, and all specimens were unilateral, histologically confirmed acoustic neuromas. All patients had undergone primary microsurgical tumour resection, except for one, who had previously relapsed after stereotactic radiosurgery.

Sections were cut at $5 \mu \mathrm{m}$ and floated on to positively charged slides (SuperFrost Plus; Menzel-Glaser, Portsmouth, New Hampshire, USA), from representative formalin fixed, paraffin wax embedded tumour blocks. The slides were dewaxed in xylene and rehydrated through graded ethanols. Heat induced epitope retrieval was carried out by immersing the slides in citrate buffer ( $\mathrm{pH} \mathrm{6}$ ) and microwaving at $600 \mathrm{~W}$ for 20 minutes, before cooling and rinsing with phosphate buffered saline (PBS). Endogenous peroxidase activity was quenched by incubating the sections in $1 \% \mathrm{H}_{2} \mathrm{O}_{2}$. Nonspecific binding sites were then blocked by preincubating with 5\% normal swine serum (Dako Ltd, Ely, Cambridgeshire, UK) and $1 \%$ bovine serum albumin (BSA) in PBS/0.3\% Triton X-100 for 20 minutes at room temperature. Sections were then incubated overnight at $4{ }^{\circ} \mathrm{C}$ with primary antibody, diluted 1/10 000 in PBS plus 1\% BSA and $0.3 \%$ Triton X-100. The rabbit polyclonal antibodies to SSTRs 1, 2, and 5 were produced and provided by Dr Helboe, as described previously. ${ }^{17}$ Raised against the C-terminal part of the human receptor proteins, the antisera have been fully characterised, and lack of crossreactivity has been demonstrated. A commercial polyclonal anti-SSTR3 antibody was used (Gramsch Laboratories, Schwabhausen, Germany), also at a dilution of $1 / 10000$.

After washing with $0.25 \%$ BSA and $0.05 \%$ Tween-20 in PBS, sections were incubated with biotinylated secondary antibody (biotinylated swine anti-rabbit IgG; E0353, Dako) at a 1/500 dilution for one hour at room temperature. Sections were then washed in PBS with $0.05 \%$ Tween-20, incubated with tyramide blocking buffer (NEN Life Science Products, Boston, Massachusetts, USA) for 20 minutes, and then incubated with horseradish peroxidase conjugated streptavidin-biotin complex (HRP-StrepABC) for 45 minutes. After rinsing in washing buffer, the signal was then amplified by applying biotinylated tyramide (DuPont NEN) at a dilution of 1/100 for 10 minutes, before a final 45 minute incubation with HRP-StrepABC. Sections were again washed, and immunoreactivity was visualised by adding $\mathrm{H}_{2} \mathrm{O}_{2}$ as enzyme substrate, in the presence of $0.05 \% 3,3^{\prime}$-diaminobenzidine. Sections were then briefly counterstained with Harris's haematoxylin, before rehydrating and mounting with DPX.
Samples of anterior pituitary and pancreas known to express the relevant antigen were used as positive controls. Negative controls included adsorption studies, as described previously, ${ }^{17}$ omission of the primary antibody, and incubation with $1 \%$ non-immune serum.

\section{QUANTIFICATION OF IMMUNOSTAINING}

Staining was graded independently by two blinded observers. Both Schwann cells and blood vessels were assessed, and cells were only regarded as positive if they showed an unequivocal brown reaction product in the cytoplasm. Because staining was uniform across the sections, it was possible to select representative areas for assessment. The immunostaining within these areas was estimated using intermediate power light microscopy $(\times 200)$. A semiquantitative scoring system was used, according to the proportion of Schwann cells or blood vessels $(0-5 \%,-; 6-25 \%,+; 26-50 \%$, $++;>50 \%,+++)$ staining positive. Positive immunostaining is defined by scores of,+++ , or +++ .

\section{RESULTS}

Table I summarises the Schwann cell staining pattern for each tumour. Each tumour expressed at least one SSTR subtype; two of the tumours expressed all four subtypes. Positive vascular staining was seen only for SSTR2, none of the other SSTR subtypes was demonstrated in blood vessels (fig 1). Overall, SSTR2 was the most prevalent subtype, being

\begin{tabular}{|c|c|c|c|c|}
\hline Tumour & SSTR I & SSTR2 & SSTR3 & SSTR5 \\
\hline 1 & + & + & ++ & - \\
\hline 2 & + & + & + & + \\
\hline 3 & - & + & - & + \\
\hline 4 & - & + & ++ & - \\
\hline 5 & - & - & + & - \\
\hline $6^{*}$ & - & + & + & + \\
\hline 7 & ++ & + & - & + \\
\hline 8 & ++ & ++ & + & + \\
\hline \multicolumn{5}{|c|}{$\begin{array}{l}\text { *The patient was treated with radiotherapy before surgery. } \\
\text { Staining was graded as follows: } 0-5 \%,-; 6-25 \%,+26-50 \%,++ \text {; } \\
>50 \% \text {, +++. } \\
\text { SSTR, somatostatin receptor. }\end{array}$} \\
\hline
\end{tabular}

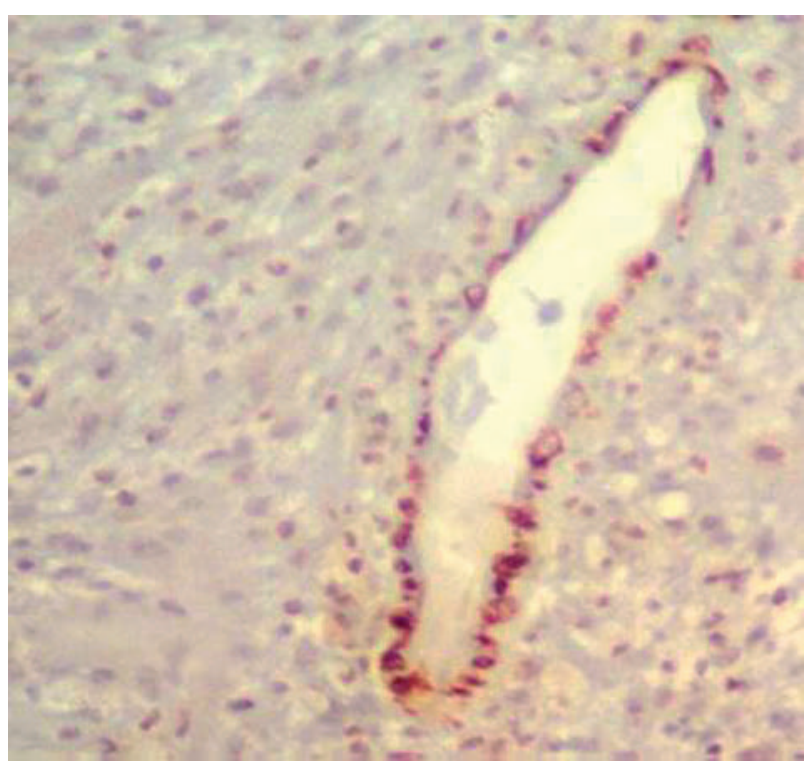

Figure 1 Positive vascular staining for somatostatin receptor 2 . 


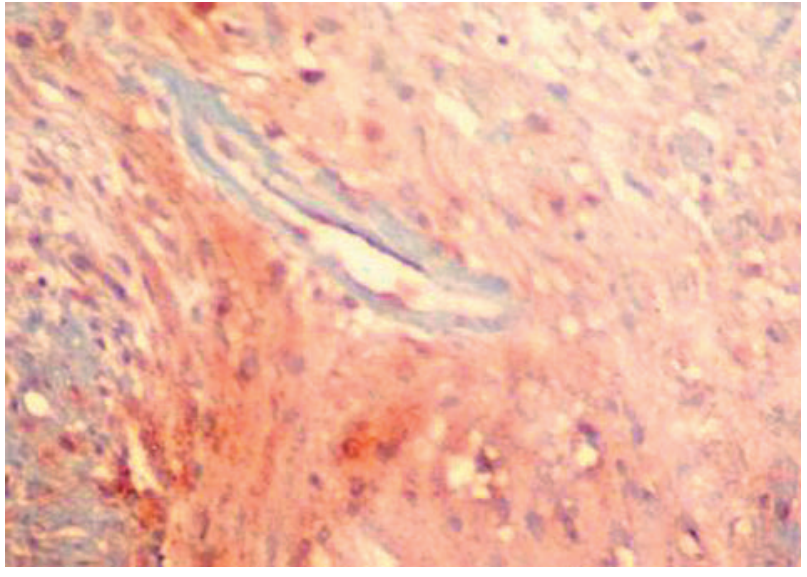

Figure 2 Schwann cells demonstrating positive staining for somatostatin receptor 3 .

expressed in the Schwann cells of seven of the eight tumours and in most of the vessels of all eight tumours. Receptor 3 was the next most prevalent, present to a low or moderate extent in six of the eight tumours studied (fig 2). There was intermediate expression of receptors 1 and 5 , in four and five of the eight tumours, respectively. No difference in receptor expression was seen between Antoni A and Antoni B areas.

\section{DISCUSSION}

Acoustic neuroma has been described as "benign in nature, but malignant in position" because of its proximity to the brainstem and cranial nerves V, VII, and VIII.

Currently, the management options for unilateral acoustic neuromas are observation, with interval scanning by magnetic resonance imaging, stereotactic radiosurgery, and microsurgical tumour resection. Both interventions have the potential for serious complications, the treatment goals being longterm control of tumour growth and preservation of neurological function. ${ }^{18} 19$ There are no reports in the literature of chemotherapy trials for acoustic neuroma. Although there have been no reports on the expression of SSTRs in acoustic neuromas, relative overexpression of SSTR2 has been reported in meningiomas, raising the possibility of SRIF analogue treatment in this condition. ${ }^{20}$ This is interesting, because these two conditions may coexist in the $10 \%$ of patients with bilateral acoustic neuromas associated with neurofibromatosis type 2. Paradoxically, however, Koper et al showed that somatostatin could stimulate the growth of cultured meningioma cells, via the inhibition of CAMP. ${ }^{21}$

\section{"Vascular expression of somatostatin receptor 2 was ubiquitous, suggesting a potential antiangiogenic role for somatostatin analogues"}

Our study shows that SSTRs 1, 2, 3, and 5 are widely and differentially expressed in acoustic neuromas. In all of the acoustic neuromas studied, there was Schwann cell expression of at least one SSTR subtype. Interestingly, receptors 2 and 3 were the most prevalent, and in other studies have caused tumour shrinkage via the inhibition of cell proliferation and induction of apoptosis, respectively. ${ }^{11}{ }^{12}$

Vascular expression of SSTR2 was ubiquitous, suggesting a potential antiangiogenic role for somatostatin analogues. There is evidence that vascular SSTRs have a direct antiangiogenic effect via the inhibition of endothelial cell growth. ${ }^{22}$ Vascular SSTRs may also have an antitumour effect

\section{Take home messages}

- Somatostain receptor 2 (SSTR2) was the most prevalent subtype in Schwann cells followed by SSTR3, then SSTR1 and SSTR5

- The vascular expression of SSTR2 was ubiquitous, but there was no evidence of SSTR 1, 3, or 5 expression in blood vessels

- SSTRs 1, 2, 3, and 5 are differentially expressed in acoustic neuromas and at least one subtype was expressed in all tumours

- Somatostatin analogues may have a therapeutic role in the management of this rare and challenging condition

through vasoconstriction, leading to tumour hypoxia and necrosis. ${ }^{1323}$

In conclusion, this is the first study to demonstrate the presence of somatostatin receptors in both the blood vessel endothelium and the Schwann cells of acoustic neuromas. This suggests that SRIF analogues may have a role in the treatment of acoustic neuromas, perhaps by causing pretreatment tumour shrinkage or by preventing tumour recurrence. These drugs are relatively non-toxic, and there is good evidence that acoustic neuromas express receptors for them. Given the variable expression of SSTRs by the tumours in our study, somatostatin analogue scintigraphy may be useful in selecting patients for SRIF analogue treatment. However, an inhibitory effect on tumour cell growth should be demonstrated before embarking on a therapeutic trial of an SRIF analogue in this condition.

\section{Authors' affiliations}

N D Stafford, L T Condon, M J C Rogers, Department of

Otolaryngology-Head and Neck Surgery, Hull Royal Infirmary, Anlaby Road, Hull HU3 2JZ, UK

L Helboe, Department of Neurobiology, H. Lundbeck A/S, Ottiliavej 7, DK-2500 Valby, Denmark

D A Crooks, Department of Neuropathology, Hull Royal Infirmary S L Atkin, Department of Diabetes and Endocrinology, Michael White Diabetes Centre, Hull Royal Infirmary

\section{REFERENCES}

1 Lanser MJ. Molecular genetics of acoustic tumours. Otolaryngol Clin North Am 1992;25:995-1009.

2 Antoni N. Ueber Ruckenmarkstumoren und Neurofibrome; Studien zur pathologische Anatomie und Embryogenese; mit einem klinische Anhang. Munich: J.F. Bergmann, 1920.

3 Brazeau P, Vale W, Burgus R, et al. Hypothalamic polypeptide that inhibits the secretion of immunoreactive pituitary growth hormone. Science 1973:179:77-9.

4 Epelbaum J, Dournaud $P$, Fodor $M$, et al. The neurobiology of somatostatin. Crit Rev Neurobiol 1994;8:25-44.

5 Stewart PM, James RA. The future of somatostatin analogue therapy. Best Pract Res Clin Endocrinol Metab 1999;13:409-18.

6 Arnold R, Simon B, Wied M. Treatment of neuroendocrine GEP tumours with somatostatin analogues: a review. Digestion 2000;62(suppl 1):84-91.

7 O'Byrne KJ, Dobbs N, Propper DJ, et al. Phase II study of RC-160 (vapreotide), an octapeptide analogue of somatostatin, in the treatment of metastatic breast cancer. Br J Cancer 1999;79:1413-18.

8 Hoyer D, Bell Gl, Berelowitz M, et al. Classification and nomenclature of somatostatin receptors. Trends Pharmacol Sci 1995; 16:86-8.

9 Benali N, Ferjoux G, Puente E, et al. Somatostatin receptors. Digestion 2000;62(suppl 1):27-32.

10 Sharma K, Patel YC, Srikant CB. C-terminal region of human somatostatin receptor 5 is required for induction of Rb and $\mathrm{Gl}$ cell cycle arrest. Mol Endocrinol 1999;13:82-90.

11 Srikant CB. Human somatostatin receptor mediated antiproliferative action evokes subtype selective cytotoxic and cytostatic signaling. Yale J Biol Med 1997; 70:541-8.

12 Sharma K, Srikant CB. Induction of wild-type p53, Bax and acidic endonuclease during somatostatin-signaled apoptosis in MCF-7 human breast cancer cells. Int J Cancer 1998;76:259-66. 
13 Reubi JC, Schaer JC, Laissue JA, et al. Somatostatin receptors and their subtypes in human tumors and in peritumoral vessels. Metabolism 1996;45:39-41.

14 Newman CB, Melmed S, Snyder PJ. Safety and efficacy of long-term octreotide therapy of acromegaly: results of a multicenter trial in 103 patients - a clinical research center study. J Clin Endocrinol Metab patients - a clinical
1995;80:2768-75.

15 O'Byrne KJ, Carney DN. Radiolabelled somatostatin analogue scintigraphy in oncology. Anticancer Drugs 1996:7(suppl 1):33-44.

16 Henze M, Schuhmacher J, Hipp P, et al. PET imaging of somatostatin receptors using $\left[\begin{array}{lll}1 & 1 & 1\end{array}\right)$ In $]$ diethylenetriaminepentaacetic-acid-octreotide (DTPAOC). J Nucl Med 2001;42:1053-6.

17 Helboe L, Moller M, Norregaard L, et al. Development of selective antibodies against the human somatostatin receptor subtypes sstl-sst5. Mol Brain Res 1997;49:82-8.
18 Ramsden RT, Moffat DA Intracanalicular acoustic neuromas: the case for early surgery. Clin Otolaryngol 1994;19:1-2.

19 Kondziolka D, Lunsford LD, Mclaughlin MR. Long-term outcomes after radiosurgery for acoustic neuromas. N Engl J Med 1998;339:1426-31.

20 Schultz S, Pauli SU, Schultz S. Immunohistochemical determination of five somatostatin receptors in meningioma reveals frequent overexpression of somatostatin receptor subtype sst2A. Clin Cancer Res 2000;6:1865-74.

21 Koper JW, Markstein R, Kohler C, et al. Somatostatin inhibits the activity of adenylate cyclase in cultured human meningioma cells and stimulates their growth. J Clin Endocrinol Metab 1992;74:543-7.

22 Albini A, Florio T, Giunciuglio D, et al. Somatostatin controls Kaposi's sarcoma tumor growth through inhibition of angiogenesis. FASEB $J$ 1999; 13:647-55.

23 Reubi JC Laissue JA. Multiple actions of somatostatin in neoplastic disease. Trends Pharmacol Sci 1995;16:110-15. 